\title{
DINÁSTICOS Y ANTIDINÁSTICOS EN LA CATALUÑA DE LA RESTAURACIÓN: Comentario a los procesos electorales
}

\author{
CONXITA MIR CURCÓ
}

\section{DEL TURNO PACÍFICO A LAS PRIMERAS DISCREPANCIAS (1876-1900)}

Partiendo de un conocimiento demasiado generalizado de los comicios de este período, podemos afirmar que la etapa restauracionista se caracteriza por una estricta observancia de los deseos del turno gubernamental en todos los niveles electorales. La normalidad registrada puede explicar el poco atractivo que este período ha ejercido en los estudiosos, circunstancia que no exime de la necesidad de analizarlo, puesto que sólo así podremos ir resolviendo los diversos interrogantes planteados en torno al carácter que adquirió el sistema de la Restauración en una Cataluña que, durante el sexenio revolucionario, había registrado una vitalidad electoral que, a partir de 1874 , vemos de repente trocada en indiferencia. Tampoco es fácilmente justificable que de la apatía puedan surgir elementos como el catalanismo, el republicanismo o el obrerismo, que en pocos años darian a Cataluña un dinamismo político y social, germen a su vez de las principales crisis políticas del régimen hasta 1923.

Cabe, no obstante, recordar que, en cuanto a fraude y desvirtuación de la práctica electoral democrática, en 1875 no se hace sino recuperar una larga tradición que ni la gloriosa revolución ni la primera República habían podido romper. El régimen de la Restauración con la reducción drástica del censo electoral -sufragio censitario- no hizo más que acentuar la falta de representatividad del proceso electoral, alejando de las urnas a los sectores populares, más numerosos y difíciles de controlar (1).

Solamente las fuerzas republicanas reclamaban por entonces un sufragio libre y universal, aunque, por las propias características del sistema, se mantenían alejadas de la lucha política, y sufrían represión ante cualquier ensayo de movilización, con la salvedad de poder acceder a algún acta de diputado dentro de la cuota de opositores que los gobiernos dinásticos admitían en las diversas legislaturas. Por otra parte, el estudio de Ángel Duarte sobre el republicanismo catalán de la década de los noventa nos muestra una

(1) No hay ningún trabajo global sobre las elecciones en Cataluña entre 1876 y 1900 . Puede consultarse una «Relació dels resultats electorals de 1869 a 1899» en la obra «Les eleccions generals a Catalunya de 1901 a 1923” de A. Balcells, J. B. Culla y C. Mir, Estudis Electorals/4. Publicaciones de la Fundació Jaume Bofill, Barcelona, 1982. 
connivencia altamente practicada entre republicanos y liberales respecto al reparto de áreas de influencia, siendo reveladores los escasos análisis efectuados sobre elecciones municipales de la época. Conocemos la temprana presencia republicana en los consistorios de Barcelona, Reus, Manresa, Figueras, Tarragona, etc., muestra de que un sector de la oposición antidinástica asumía la estrecha vía de participación política que el sistema canovista le reservaba, convencido de poder luchar contra el régimen desde el interior de sus instituciones, o persuadido de que sólo desde el poder era posible obtener beneficios para la causa (2).

Con todo, el dilema participación-retraimiento persistía en los debates entre las fuerzas republicanas, incluso después de haberse ampliado el margen de maniobra con la nueva ley electoral de 1890 , que reintroducian el sufragio universal masculino. Los éxitos republicanos en elecciones legislativas siguieron siendo escasos y muy localizados: posibilistas y republicanos con prestigio local -Sol i Ortega, Vallés i Ribot_- o nacional - Pi i Margall- iban, infravalorando el descrédito que esta conducta les generaría a corto término, turnándose los distritos o repitiendo candidatura en más de uno de ellos, para así asegurar su presencia en las Cortes.

Para acabar, si poco es lo que conocemos sobre las relaciones electorales entre el turno dinástico y la oposición republicana, menos sabemos aún sobre los primeros pasos político-electorales del catalanismo antes del acto fundacional de la Lliga Regionalista en 1901. Tomando como ejemplo el caso de Reus, se sabe que ya en 1887 había una representación de filiación catalanista en el Ayuntamiento de aquella ciudad, circunstancia que sugiere la posibilidad de una más temprana incorporación del catalanismo a las luchas políticas del país. En cualquier caso, no será hasta la presentación de la candidatura catalanista «dels quatre presidents» en las elecciones legislativas del distrito de Barcelona ciudad en mayo de 1901 que se iniciará en Cataluña la sustitución del turno liberal-conservador por otro regionalistarepublicano.

\section{EL TURNO INTERFERIDO (1901-1923)}

\section{La ciudad de Barcelona}

El triunfo electoral de la oposición antidinástica en Barcelona se renovó en noviembre del mismo 1901, cuando republicanos y regionalistas vencieron a los monárquicos en las elecciones municipales. Roto el turno dinástico en las elecciones de aquel año, ni conservadores ni liberales lograrían nunca

(2) Ver Ángel Duarte, «El republicanisme català a la fi del segle XIX», Eumo Editorial. Vic, 1987. 
la recuperación de su hegemonía en ninguno de los tres niveles -municipal, provincial o general- en los que se celebraban los comicios.

El Ayuntamiento de Barcelona quedaría en manos de los republicanos hasta 1915, siendo los radicales seguidores de Alejandro Lerroux la fuerza mayoritaria (ver cuadro número 1), mientras la Lliga Regionalista, aliada con carlistas y monárquicos autonomistas, se hace con el control del consistorio desde ese momento hasta la Dictadura de Primo de Rivera, siendo también durante dicha época la fuerza mayoritaria de la Mancomunitat de Catalunya. El reemplazo del turnismo liberal-conservador por el regionalista-republicano se había consumado (3).

\section{CUADRO 1}

\section{ELECCIONES MUNICIPALES EN LA CIUDAD DE BARCELONA N. ${ }^{\circ}$ de Concejalías}

\begin{tabular}{|l|rrrrrrrrrrr|}
\hline $\begin{array}{c}\text { ELECCIONES } \\
\text { FILIACIÓN }\end{array}$ & 1901 & 1903 & 1905 & 1907 & 1909 & 1911 & 1913 & 1915 & 1917 & 1920 & 1923 \\
\hline Monárquicos & 4 & & & & & & 1 & 3 & & 4 & \\
\hline Carlistas & & & & & & & 1 & 1 & 1 & 2 & 2 \\
\hline Regionalistas & 11 & 7 & 12 & 4 & 5 & 11 & 11 & 9 & 13 & 14 & 15 \\
\hline Republicanos & 10 & 18 & 14 & 24 & 20 & 17 & 12 & 12 & 12 & 6 & 8 \\
Radicales & & & & 16 & 14 & 11 & 11 & 9 & 11 & 5 & 7 \\
Nacionalistas & & & & 8 & 6 & 6 & 1 & 2 & 1 & & \\
Independientes & & & & & & & & & & &
\end{tabular}

FUENTE: Elaboración propia a partir de I. Molas, Lliga Catalana, Vol. I; dossier "L'ajuntament de Barcelona al segle XX», L’Avenç n. ${ }^{\circ} 58$, marzo 1983; J. B. Culla, El Republicanisme Lerrouxista a Catalunya (1901-1923).

(3) Sobre las elecciones municipales de la ciudad de Barcelona además de la bibliografía con la que se ha elaborado el cuadro número 1, pueden consultarse los trabajos de James Ronald Kelly, "Les eleccions municipals i parlamentaries als districtes de Barcelona entre $1910 i$ 1923» tesis de licenciatura, Universidad de Barcelona (1980) y "A neighbourhood approach to working class life and politics in Barcelona, 1910-1923", tesis doctoral, Universidad de Barcelona, 1982. «Les eleccions legislatives i municipals a Barcelona, 1810-1986. Context politice, resultats electorals» i «Eleccions de diputats provincials a Barcelona, 1812-1923", Ajuntament de Barcelona, Fundació Jaume Bofill, Col.lecció Atles Estadistic de Barcelona, Temas Electorals 213, 1989. Sobre las elecciones a la Diputación provincial de Barcelona, podrá consultarse la "Historia de la Diputación de Barcelona", tercer volumen, bajo la coordinación de Borja de Riquer, de próxima aparición. Rosa Torán y Celia Cañellas, «La clase política barcelonina 1:8751901», a "Actituds politiques: control social a la Catalunya de la Restauració (1875-1923)», Lleida, 1989. 
Los resultados de las elecciones legislativas de 1901 (ver cuadro número 2) presentan una coherencia bastante estrecha con los de las municipales, testimonio, posiblemente, de la depuración del proceso electoral en las grandes ciudades. Siendo la Lliga el partido inicialmente mayoritario - tanto en las legislativas como en las municipales de 1901- en Barcelona, cede más tarde el terreno a los republicanos, para recuperarse paulatinamente después de 1910, llegar a ser el partido mejor situado en las legislativas de 1914 y en las municipales de 1917, y no abandonar esta posición hasta los albores de la Dictadura.

\section{CUADRO 2}

\section{RESULTADOS DE LAS ELECCIONES LEGISLATIVAS EN LA CIUDAD DE BARCELONA (\% de votos sobre el censo electoral)}

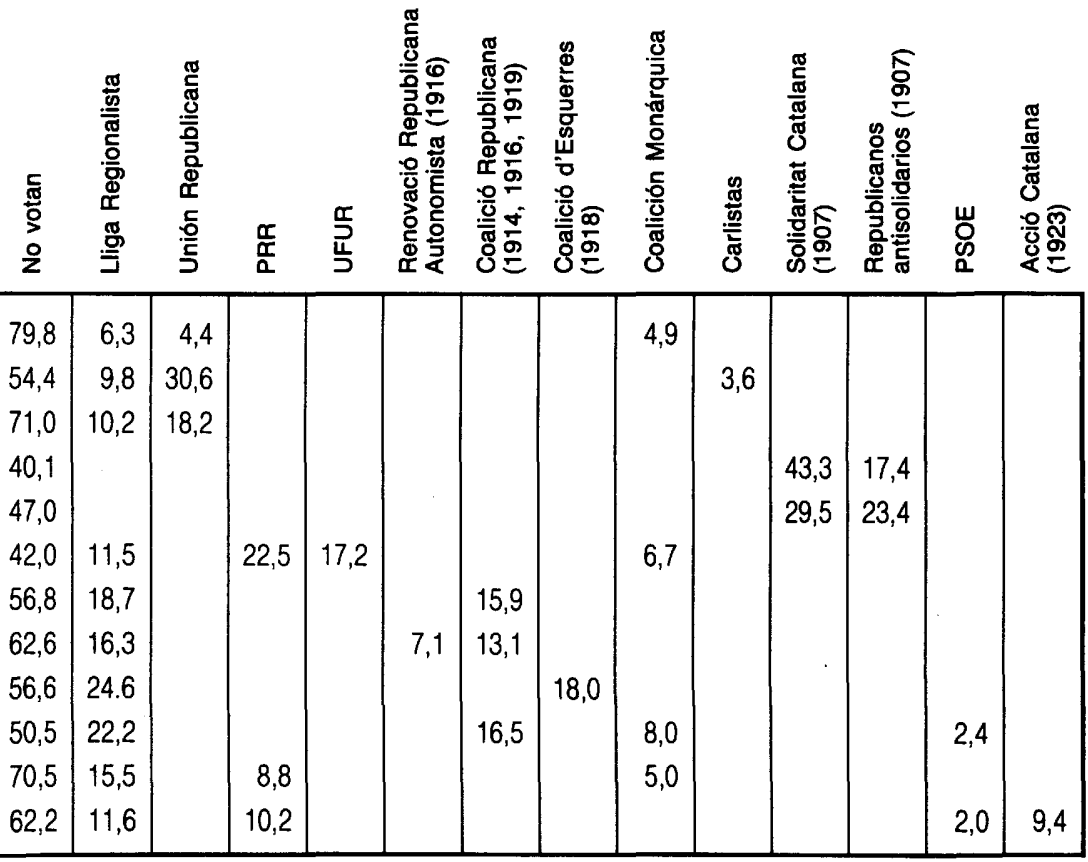

FUENTE: Reproducción de A. Balcells, J. B. Culla y C. Mir Les eleccions generals a Catalunya de 1901 a 1923.

Desde 1918 la Lliga incluye en sus listas un candidato carlista, que sale elegido hasta 1923 , sin que se haga distinción de filiación.

El Partido Republicano Radical (PRR) presenta candidatura por minorías con dos candidatos en las elecciones de 1920 y 1923.

La Coalició d'Esquerres de 1918 estaba formada por: Partit Republicà Català, Partit Republicà Radical y Partido Socialista Obrero Español. Este último presenta candidatura por minorías los años 1919 y 1923.

El cuadro no recoge ni las candidaturas individuales con pocos votos, ni los votos en blanco o nulos. 
Los republicanos conocieron su apogeo electoral en las elecciones a Cortes de 1903 y entre las de 1907 - de la Solidaritat Catalana-y 1914. Divididos entre radicales y nacionalistas no consiguieron, ni sumando sus influencias respectivas, prolongar más allá su predominio en el reparto de escaños parlamentarios o municipales. Es pues hacia el final de la segunda década del siglo, coincidiendo con la agudización de la crisis social, cuando las calles y las fábricas de Barcelona registran una situación que no es exagerado calificar de prerrevolucionaria - revolución de 1917, huelgas de 1919, pistolerismo, etc...- que el republicanismo en su manifestación político-electoral declina notablemente, con sus líderes situados ante el dilema de la inoperancia o la clandestinidad.

Más interesante que la descripción de la ruptura del turno dinástico en Barcelona, al fin y al cabo poco significativa en el cómputo global de actas de las Cortes, quizá sea resaltar el aspecto cualitativo de este cambio político: por una parte es posible, probablemente por vez primera en el pais, que el cuerpo electoral se exprese libremente y que los escrutinios reflejen esta expresión. En otro orden aparece con fuerza lo que diversos autores han dado en llamar el sistema moderno de partidos, semejante al imperante en otros países europeos con democracias más formales y más reales que la esparĩola.

La primera cuestión, la de la participación electoral real, hay que considerarla, una vez roto el turno en la Barcelona de 1900, dentro del margen de maniobra que el régimen dinástico estaba dispuesto a conceder a la oposición. Era mucho más fácil, barato y, especialmente, quedaba más impune, cometer tropelías electorales por apartados distritos rurales que por las calles de las grandes ciudades.

El nuevo sistema de partidos que se configura en Cataluña desde principios de siglo es, en primer término, un sistema de encuadramiento social de una colectividad muy activa y a la vez conflictiva. La Lliga Regionalista viene a cubrir el espacio vacío que existe para un partido conservador de corte europeo, que defienda los intereses de una pujante burguesía industrial, financiera y comercial de un modo efectivo. $Y$ no es que los partidos dinásticos no tuvieran dicha vocación, pero les faltaba un elemento aglutinador que además de vencer les permitiera convencer. En Cataluña este elemento no era otro que el catalanismo, término que engloba a cualquier afán por defender las peculiaridades lingüisticas, culturales y económicas de la sociedad catalana. Este primer tipo de catalanismo político organizado aparece como signo del dinamismo de la burguesía catalana y no hace apenas cuestión del conflicto social (la prosperidad del obrero está en la de su patrón) que subyace en una Barcelona donde es posible ver cómo crece la hierba cada día. La clase obrera barcelonesa, sufriendo aún las consecuencias de la crisis que 
siguió al Desastre, pero esperanzada en la reindustrialización del país, está al inicio del siglo XX huérfana de líderes y organizaciones, aparte de grupúsculos anarquistas.

Es en un momento así cuando irrumpe Lerroux, con un verbo, aunque demagógico, sencillo y cautivador para los oídos de los humildes, con sus Casas del Pueblo, bailes, y «meriendas populares» multitudinarias. Un sucedáneo de partido de clase cuya maquinaria política y electoral funcionó con pleno rendimiento durante más de 15 años, dejando para la posteridad el término «lerrouxismo", hoy utilizado en sentido peyorativo contra todo aquel que desde dentro de Cataluña se manifiesta como anticatalanista, aunque esta interpretación actual es, por simplificadora, fundamentalmente errónea (4).

Hasta las elecciones de 1907, las de la Solidaridad, fueron estas dos formaciones las que marcaron la pauta política de la capital del principado, mientras un tercer grupo, germinado a partir de una disidencia progresista de la Lliga y enriquecido con republicanos de ideología nacionalista, pugnó por atraerse el apoyo de la media y pequeña burguesía, desde postulados republicanos y catalanistas. Este grupo, encuadrado en 1910 en la UFNR, se iría escindiendo después en diferentes partidos progresivamente más radicalizados y cada vez con menor incidencia electoral.

Fuera de estas tres formaciones y de sus derivados asimilables sólo cabe citar una leve pero constante presencia de restos del tradicionalismo y de los partidos dinásticos en el Ayuntamiento y en la Diputación, que se acentúa a medida que las fuerzas republicanas van perdiendo peso en favor de las regionalistas.

Lástima que el pueblo barcelonés no estuviera para elecciones. Pues considerando incluso la democratización del escrutinio y la existencia de una actividad política real promovida por partidos con vocación parlamentaria, los graves problemas que estallaban en la ciudad y en el país en general se dilucidaban lejos de las urnas. La burguesía barcelonesa se vio muy tempranamente en la contradicción de apoyar electoralmente opciones supuestamente democráticas como la Lliga y aprobar la represión del ejército contra las masas que ocupaban las calles, como sucedió durante la semana trágica de 1909, o durante la crisis de 1917. El corolario final de esta situación fue que el régimen se vio en la imposibilidad de canalizar la opinión popular mediante el sufragio, y la clase obrera desbordó en sus reivindicaciones y acciones incluso a sus propios dirigentes.

(4) Sobre el papel de A. Lerroux en Cataluña es de consulta obligada la obra de Joan B. Culla i Clarà «El republicanisme lerrouxista a Catalunya (1901-1923)». Curial. Barcelona, 1986. 


\section{El resto de Cataluña}

Pero Cataluña ha mirado siempre e inevitablemente hacia su capital. Piénsese que la segunda ciudad del principado a principios de siglo, Reus, sólo albergaba una población equivalente a la vigésima parte de la de Barcelona, que por entonces sobrepasaba el medio millón de habitantes. Por ello, el modelo de cambio político-electoral barcelonés se intentó importar e imitar en los demás distritos electorales catalanes, rurales la mayoría de ellos, aunque fuese con retardo. La extensión de la influencia barcelonesa fue, en efecto, paulatina y con matices, siendo el primero de ellos la posición de relativa importancia que las fuerzas dinásticas pudieron mantener fuera de Barcelona hasta el final del período (5).

Durante este tiempo se distribuyeron en los distritos uninominales de la provincia de Barcelona 141 escaños, de los cuales 85 fueron para los partidos antidinásticos y 56 para los del turno (ver cuadro número 3). Se destaca la Lliga Regionalista como primera fuerza en esta zona, por encima del conjunto de todas las opciones republicanas. La ruptura del turno no fue nunca absoluta, produciéndose el cambio de orientación en el reparto de los escaños en favor de los antidinásticos hacia el 1905, año en que las fuerzas dinásticas solamente consiguen 6 de las 13 actas en juego.

También en la provincia de Gerona el saldo es favorable a los antidinásticos -53 actas frente a 35-, siendo igualmente la Lliga Regionalista la primera fuerza en liza, aunque en estos distritos los republicanos alcanzarán unos buenos resultados. De las once elecciones del período sólo en tres los dinásticos obtienen mayoria de actas. A partir de 1907, a pesar de mantenerse reductos monárquicos importantes, el turno es netamente republicanoregionalista.

(5) Además de la obra de carácter general de A. Balcells, J. B. Culla y C. Mir «Les eleccions generals a Catalunya 1901-1923», Publicacions de la Fundació J. Bofill. Estudis electorals/4, Barcelona 1982, pueden consultarse también los estudios monográficos de $\mathrm{C}$. Mir, " $\mathrm{Ca}$ ciquisme polític i lluita electoral, Lleida 1890-1936”, Publicacions de l'Abadia de Montserrat, Barcelona, 1985; Jaquim Molins, «Elecciones y partidos en la provincia de Tarragona, 1890-1936», Publicacions de la Diputació de Tarragona, 1985; Josep Cllara, "El personal polític a l'Ajuntament de Girona (1917-1987), Cercle d'Estudis Històrics i Socials, Girona, 1987; Antoni Gustems, «Les eleccions provincials a Girona, 1901-1923, Tesis de Licenciatura, Universidad Autònoma de Barcelona. 


\section{DISTRITOS ELECTORALES DE CATALUÑA (1901-1923)}

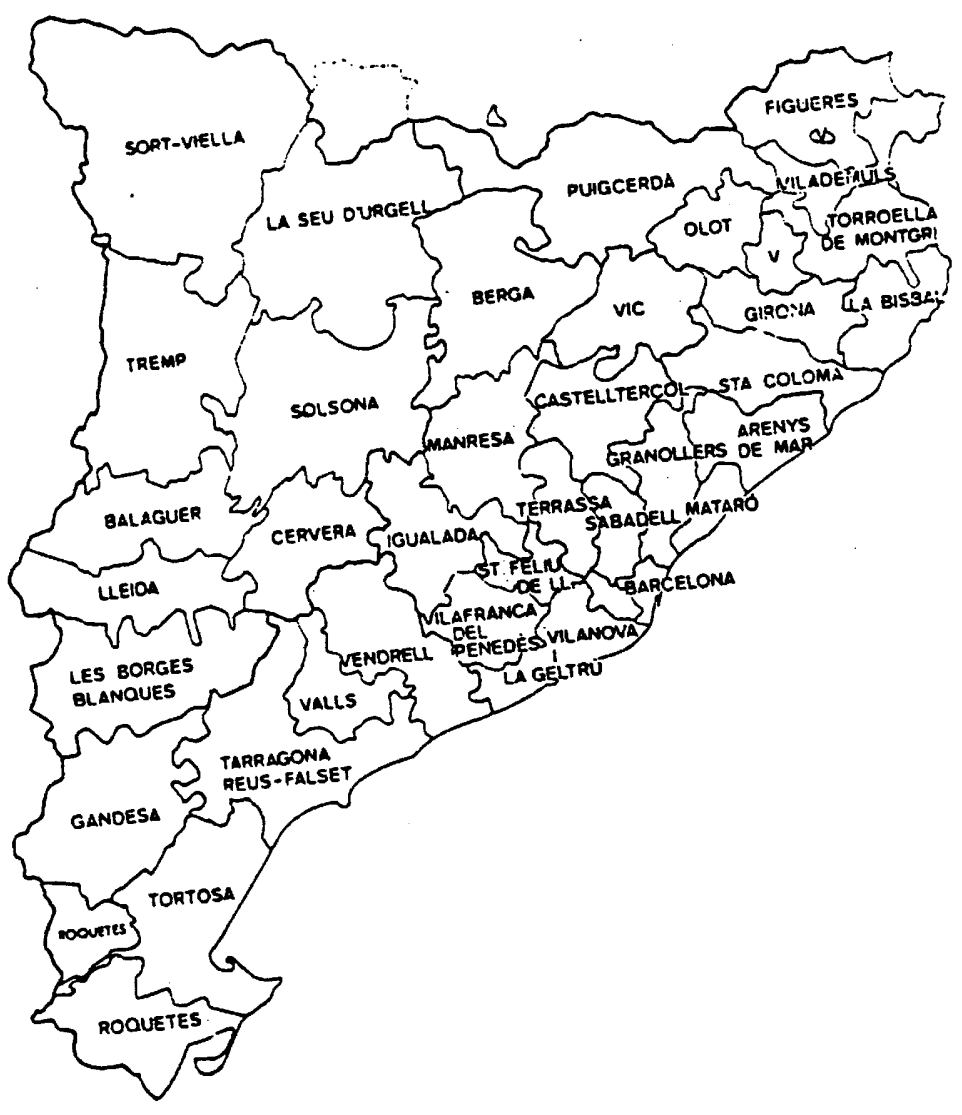

Fuente: Reproducido de A. Balcells, J. B. Culla y C. Mir, Les eleccions generals a Catalunya de 1901 a 1923. 
CUADRO 3

BARCELONA - PROVINCIA

ELECCIONES LEGISLATIVAS 1901-1923

N. ${ }^{\circ}$ DE ESCAÑOS OBTENIDOS EN TODO EL PERÍODO

\begin{tabular}{|c|c|c|c|c|c|c|c|c|c|c|c|}
\hline & MC & ML & UMN & FMA & $\begin{array}{c}\text { Total } \\
\text { Dinast. }\end{array}$ & $\mathbf{T}$ & LL & $\mathbf{I}$ & $\mathbf{R}$ & Rf. & $\begin{array}{c}\text { Total } \\
\text { Antl-Din } \\
\end{array}$ \\
\hline Arenys de Mar & 6 & & 1 & 2 & 9 & & 2 & & & & 2 \\
\hline Berga & 1 & 2 & 3 & & 6 & 1 & 3 & 1 & & & 5 \\
\hline Castellterçol & 3 & & & & 3 & & 8 & & & & 8 \\
\hline Granollers & 1 & 2 & & & 3 & & 8 & & & & 8 \\
\hline Igualada & 3 & 4 & 1 & 1 & 9 & & 2 & & & & 2 \\
\hline Manresa & 2 & & & & 2 & & 9 & & & & 9 \\
\hline Mataró & & 5 & 2 & & 7 & & 4 & & & & 4 \\
\hline Sabadell & 2 & & & & 2 & & 1 & & 8 & & 9 \\
\hline St. Feliu de Llobregat & & 2 & & & 2 & & 5 & & 3 & 1 & 9 \\
\hline Terrassa & & 6 & 3 & & 9 & & & & 2 & & 2 \\
\hline Vic & 2 & 2 & & & 4 & 3 & 4 & & & & 7 \\
\hline Vilafranca del Penedès & & 1 & & & 1 & & 1 & & 4 & 3 & 10 \\
\hline Vilanova i la Geltrú & & 1 & & & 1 & & 9 & & 1 & & 10 \\
\hline TOTAL & 20 & 25 & 10 & 3 & 58 & 4 & 56 & 1 & 18 & 6 & 85 \\
\hline
\end{tabular}

MC: monárquicos conservadores, ML: monárquicos liberales, UMN: Unión Monárquica Nacional, FMA: Federación Monárquica Autonomista, T: tradicionalistas, LL: Lliga Regionalista, I: independientes, R: republicanos, Rf: reformistas

FUENTE: Reproducido de A. Balcells, J. B. Culls, y C. Mir, Les eleccions generals a Catalunya de 1901 a 1923.

CUADRO 4

GERONA. ELECCIONES LEGISLATIVAS 1901-1923

N.` DE ESCAÑOS OBTENIDOS EN TOdO EL PERÍOdO

\begin{tabular}{|lcccc|c|cccc|c|}
\hline & MC & ML & UMN FMA & $\begin{array}{c}\text { Total } \\
\text { Dinast. }\end{array}$ & T & LL & R & Rf. & $\begin{array}{c}\text { Total } \\
\text { Anti-Din. }\end{array}$ \\
\hline Girona & 1 & 1 & 1 & 2 & 5 & 1 & 1 & 4 & & 6 \\
La Bisbal & & 2 & & & 2 & & & 9 & & 9 \\
Figueres & & 1 & 1 & & 2 & & & 9 & & 9 \\
Olot & & & 2 & & 2 & 4 & 5 & & & 9 \\
Puigcerdà & 3 & & & & 3 & & 7 & & 1 & 8 \\
St. Coloma de Farners & 1 & 2 & & & 3 & & 8 & & & 8 \\
Torroella de Montgrí & 8 & & 2 & & 10 & & 1 & & & 1 \\
Vilademuls & & 6 & 2 & & 8 & 1 & 2 & & & 3 \\
\hline TOTAL & 13 & 12 & 8 & 2 & 35 & 6 & 24 & 22 & 1 & 53 \\
\hline
\end{tabular}

FUENTE: Reproducido de A. Balcells, J. B. Culls, y C. Mir, Les eleccions generals a Catalunya de 1901 a 1923. 
En la provincia de Lérida el equilibrio dinásticos-antidinásticos es ligeramente favorable a los primeros -47 contra 41 escaños-, gracias a la falta de fisuras antes de $1907 \mathrm{y}$ al continuado predominio de liberales y conservadores en cuatro de los ocho distritos. A diferencia de Barcelona y Gerona, en Lérida es la oposición republicana la relativamente mejor situada. Liberales, republicanos y regionalistas, son, por este orden, las fuerzas directoras en los distritos leridanos.

\section{CUADRO 5}

\section{LÉRIDA. ELECCIONES LEGISLATIVAS 1901-1923} N. ${ }^{\circ}$ DE ESCAÑOS OBTENIDOS EN TODO EL PERIODO

\begin{tabular}{|c|c|c|c|c|c|c|c|c|c|c|c|}
\hline & MC & ML & UMN & FMA & \begin{tabular}{|c|} 
Total \\
Oinast.
\end{tabular} & $\mathbf{T}$ & $\begin{array}{l}\text { LLI } \\
\text { IRg }\end{array}$ & $\begin{array}{l}\mathbf{R} / \\
\mathbf{R N} \\
\end{array}$ & Rf. & $\mathbf{N I}$ & $\begin{array}{c}\text { Total } \\
\text { Anti-Din. }\end{array}$ \\
\hline Lérida & 2 & 2 & & & 4 & & & 6 & 1 & & 7 \\
\hline Balaguer & 2 & 1 & & & 3 & & 4 & 4 & & & 8 \\
\hline Borges Blanques & 1 & 2 & & & 3 & & 1 & 4 & & 3 & 8 \\
\hline Cervera & & 7 & 1 & 1 & 9 & 1 & 1 & & & & 2 \\
\hline La Seu d'Urgell & 3 & 3 & 1 & & 7 & & 4 & & & & 4 \\
\hline Solsona & 3 & 3 & 1 & & 7 & & 4 & & & & 4 \\
\hline Sort-Viella & & 8 & 2 & & 10 & & 1 & & & & 1 \\
\hline Tremp & & 3 & 1 & & 4 & & 1 & 2 & 4 & & 7 \\
\hline TOTAL & 11 & 29 & 6 & 1 & 47 & 1 & 16 & 16 & 5 & 5 & 41 \\
\hline
\end{tabular}

IRg: Independientes regionalistas, RN: republicanos nacionalistas, $\mathrm{Nl}$ : nacionalistas independientes FUENTE: Reproducido de A. Balcells, J. B. Culls, y C. Mir, Les eleccions generals a Catalunya de 1901 a 1923.

\section{CUADRO 6}

TARRAGONA. ELECCIONES LEGISLATIVAS 1901-1923 N. ${ }^{\circ}$ DE ESCAÑOS OBTENIDOS EN TODO EL PERÍODO

\begin{tabular}{|lrrr|r|rrrr|r|}
\hline & MC & ML & UMN & $\begin{array}{c}\text { Total } \\
\text { Dinast. }\end{array}$ & T & LL & R & Rt. & $\begin{array}{c}\text { Total } \\
\text { Anti-Din. }\end{array}$ \\
\hline Tarragona-circunscripción & 4 & 8 & 3 & 15 & 2 & 2 & 14 & & 18 \\
Gandesa & & 4 & & 4 & & 1 & 5 & 1 & 7 \\
Roquetes & 2 & 7 & 2 & 11 & & & & & \\
Tortosa & 2 & 3 & 1 & 6 & & & 5 & & 5 \\
Valls & 2 & 5 & 1 & 8 & & 2 & 1 & & 3 \\
Vendrell & 2 & 4 & & 6 & & & 5 & & 5 \\
\hline TOTAL & 12 & 31 & 7 & 50 & 2 & 5 & 30 & 1 & 38 \\
\hline
\end{tabular}

FUENTE: Reproducido de A. Balcells, J. B. Culls, y C. Mir, Les eleccions generals a Catalunya de 1901 a 1923. 
Tarragona, socioelectoralmente más próxima a Lérida que a Barcelona y Gerona, aparece con una hegemonía globalmente dinástica, de corte netamente liberal, y con una implantación republicana muy considerable. La ausencia de los regionalistas - sólamente 5 escaños repartidos de forma irregular por diferentes distritos- es quizá la característica diferencial más notable de esta zona. Solamente en 2 de los 6 distritos los antidinásticos llegaron a reunir más actas que los gubernamentales y ni siquiera en 1907 hay una ruptura total con el hábito de investir turnos, pues dos de las únicas tres actas dinásticas de aquel año fueron obtenidas en distritos tarraconenses.

Una visión global de los resultados provinciales muestra que fuera de la ciudad de Barcelona las fuerzas dinásticas siguieron bien instaladas hasta el final del período. Solidaritat Catalana impulsó la movilización electoral en algunos distritos, dándose desde la oposición nuevas perspectivas a unos electores poco habituados a presenciar cambios políticos significativos. A su vez Solidaritat potenció las pequeñas fuerzas políticas locales, especialmente republicanas, que obtuvieron relevancia suficiente para conseguir voz y voto en las decisiones de las organizaciones centrales de Barcelona, que a partir de este momento empiezan a coordinar las vicisitudes de las luchas electorales en todo el ámbito catalán. Coordinación que llevó aparejada la designación de candidatos afines, a menudo enviados desde la capital. También la experiencia solidaria supuso un cambio de formas, de manera de hacer los comicios, que ya no se abandonaría. Después de 1907, dinásticos y antidinásticos hubieron, como mínimo, de guardar las formas democráticas en la mayoría de los distritos catalanes. Sin embargo hay que recordar, también, que si antes de 1907 era muy común que un diputado se instalase indefinidamente en un distrito, de modo que en el encasillado previo a las elecciones los partidos dinásticos respetaban un cupo para candidatos considerados irrevocables, la entrada beligerante de los partidos de la oposición en el juego electoral no hace varias la norma, en contra de lo esperable de un concepto más político de lo que debe ser una representación de un distrito.

Esta contradicción supuso que la articulación de un nuevo sistema de partidos, de apariencia más democrática, se hiciera con la presencia en su seno de un aparato neocaciquil que permite a la oposición ganar unos reductos electorales que antes fueron dinásticos. Republicanos y regionalistas aceptan implícitamente que, ante un electorado indiferente, bueno es cortejar a las fuerzas vivas de cada demarcación que saben, y disponen de recursos, cómo ganar un acta.

Sin duda el sistema de la Restauración tuvo una capacidad tampón -en el sentido químico del término- muy amplia, que le permitió absorber tendencias creadoras de desequilibrios cambiando muy poco de esencia. En al- 
gún momento los partidos dinásticos vieron que Catalunya se les iba de las manos, más por defección del caciquismo que por pérdida de unos votos, a fin de cuentas ficticios. En lugar de usar la máquina represora optaron por derivar esfuerzos a zonas de España más controlables. Lo cierto es que las mayorías parlamentarias dinásticas no estuvieron jamás comprometidas.

\section{Cataluña en el contexto electoral español}

Los distritos catalanes aportaban aproximadamente el $11 \%$ de los diputados españoles. Su peso específico electoral es bajo - no así el demográfi$\mathrm{Co}$, pero éste es un problema que también afecta a Madrid, Valencia, Zaragoza, etc. He aquí pues una de las claves para interpretar el grado de flexibilidad de los gobiernos dinásticos hacia la acción electoral de la oposición catalana.

El cuadro número 7 es harto revelador del contraste que aquí se pretende ilustrar: en ninguna de las elecciones generales habidas de 1901 a 1923 Cataluña llega a pagar su "cuota» de diputados dinásticos, que representan siempre en torno al $85 \%$ de la composición final del Congreso. Por el contrario, siempre supera con creces ese $15-20 \%$ de diputados no dinásticos con que suele contar la Cámara. Abundando más: sólo hasta 1907 los diputados catalanes dinásticos son más que los de la oposición. A partir de la Solidaritat nunca llegarán los primeros siquiera a conquistar la mitad de los escaños. Cierto que a partir de esta fecha en España también se produjo un progreso en la representatividad de la oposición -pasa del $12 \%$ en 1905 al $18 \%$ en 1907 y al 17\% en 1910-, progreso que en el caso que nos ocupa no es sino un artefacto matemático, ya que se debe exclusivamente al caso catalán.

A lo largo del período, y cualesquiera que sean las oscilaciones las oposiciones, siguen siendo siempre sorprendentemente estables los porcentajes estatales de representación parlamentaria para dinásticos y antidinásticos: algo más del $80 \%$ los unos, algo menos del $20 \%$ los otros. Demasiado estables como para no adjudicarles una intencionalidad, una distribución previa, por lo demás de todos conocida: el encasillado. Por lo tanto, y en lo que a mayorías parlamentarias se refiere, el Régimen de la Restauración no sólo no estuvo en peligro en ningún momento, sino que incluso se permitió mantener en su seno un foco regional de oposición permanente, del mismo modo que consintió el mismo fenómeno en las grandes ciudades del país, merced al mecanismo de sobrada compensación con que el régimen contaba en los distritos rurales. 


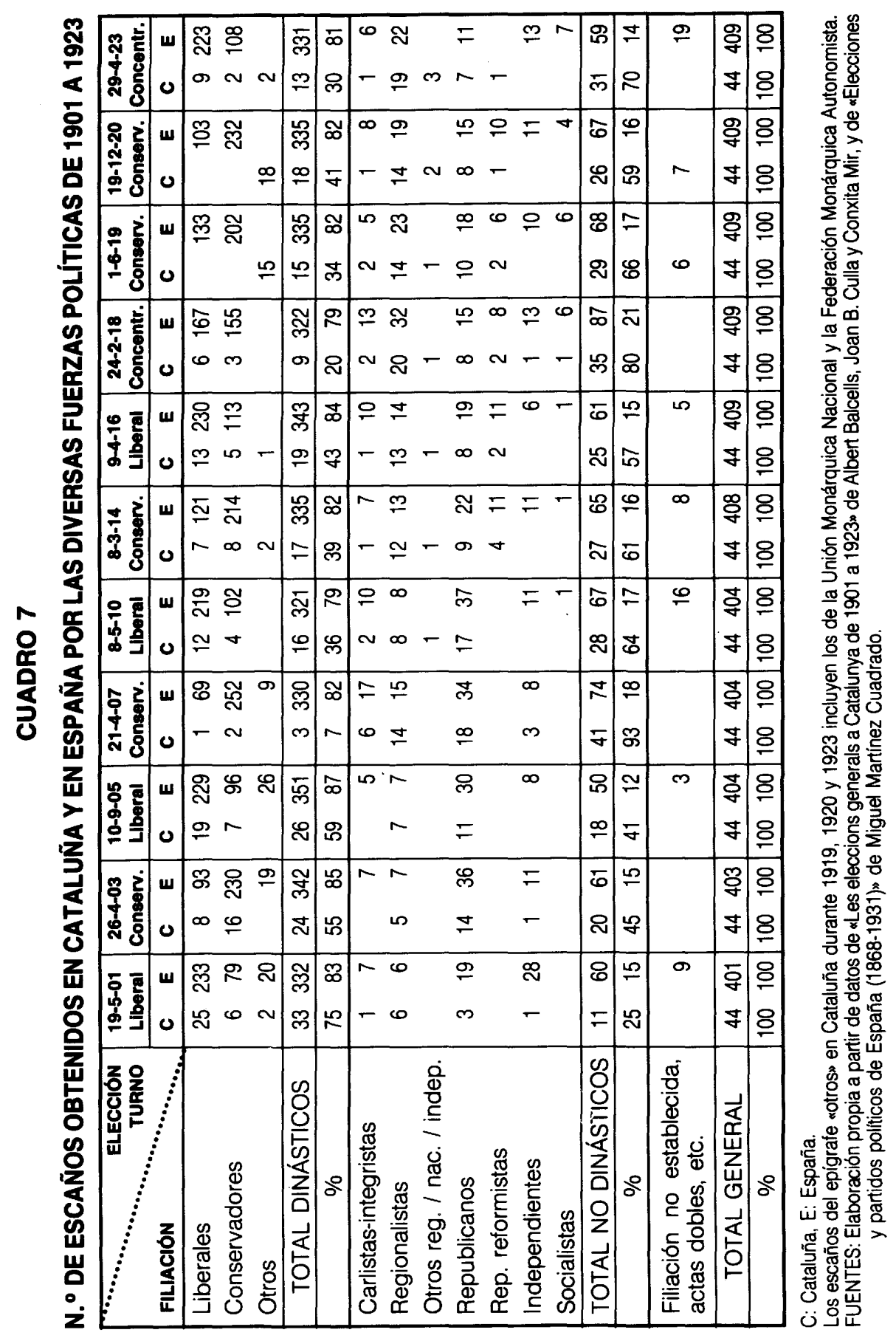


Las relaciones entre el gobierno del Estado y las fuerzas políticas catalanas no siempre fueron tensas. Incluso cristalizaron en ocasiones en pactos de carácter general, como los gobiernos de concenctración en los que la Lliga Regionalista fue llamada a participar, u operaciones de menor alcance, siendo frecuente que los partidos dinásticos, en sus relaciones en las provincias o en los distritos, promuevan o acepten alianzas coyunturales con cualquiera de las fuerzas presentes en el espectro político electoral, llegando a practicarse a lo largo del tiempo prácticamente todas las combinaciones coaligacionistas posibles. Esta circunstancia es un elemento de distorsión en cualquier análisis lineal de la evolución político-electoral de la Cataluña de la Restauración, y nos muestra que el ejemplo de la ciudad de Barcelona, donde se desarrolla un ambiente político más moderno, más democrático y más occidentalizado, no es homologable punto por punto al resto de Cataluña. Son precisos aún muchos trabajos sobre áreas reducidas con desarrollos históricos específicos para poder explicarnos la verdadera incidencia de los elementos de cambio aparecidos a lo largo del período, y el peso real de las persistencias o resistencias a este cambio.

Algo se va haciendo al respecto, aunque menos de lo deseable, dado que aún son muchas las energías consumidas en estudios comarcales o localistas desligados de un modelo explicativo global, y, por lo tanto, poco útiles para la ciencia histórica.

En torno al estudio de las interrelaciones entre los elementos de persistencia y cambio en la sociedad catalana de finales del siglo XIX y principios del XX en áreas reducidas, y por tanto con realidades más abarcables, cabe citar en primer lugar el trabajo del profesor Gabriele Ranzato sobre el distrito barcelonés de Sabadell, aparecido con el título bien explícito de «La aventura de una ciudad industrial. Sabadell entre el Antiguo Régimen y la Modernidad" (1987), en donde se aplica a analizar el momento histórico de la coexistencia entre lo viejo, los modos del Antiguo Régimen, y lo nuevo, una pujante sociedad burguesa. Ranzato nos revela hasta qué punto no es válido para el Sabadell industrial de las últimas décadas del XIX un modelo tan generalmente aceptado como el de la afinidad de la burguesía industrial por las ideas liberales y las formas políticas democráticas. Cotejando múltiples fuentes el autor nos va introduciendo paulatinamente en la manera en que se produjo la compenetración de las dos sociedades y, en el terreno político electoral, va constatando cómo las fuerzas vivas de Sabadell fueron buenas valedoras de la continuidad de un régimen fosilizado, de raíces agrarias y aristocráticas, promoviendo o apoyando opciones políticas y electorales en general, gubernamentales, reclamando como contrapartida la protección del mercado de hiladuras y tejidos contra la competencia extranjera. 
Otras contradicciones se han descritu en otros estudios apenas iniciados, como el que nos presenta Francesc Sales en su tesis de licenciatura centrada en la comarca leridana de Les Garrigues - distrito electoral de les Borges Blanques-, que nos permite observar a través del estudio de la evolución de la estructura de la propiedad de la tierra de una zona eminentemente agricola desde mediados del siglo XIX hasta la Segunda República, y a través del análisis de la coyuntura económica registrada durante el primer tercio del siglo $X X$, cómo se va gestando un sistema de interrelaciones que nos ayudan en gran manera en la comprensión de la compleja sociedad catalana de este período.

El autor reconstruye una distribución de la propiedad de la tierra basada en la desigualdad y la penuria, con predominio absoluto de la pequeña propiedad -el $80 \%$ de los propietarios poseen menos de $10 \mathrm{Ha}$. y ocupan un $30 \%$ de la tierra cultivada - que es explotada practicando el policultivo de autoabastecimiento familiar. A finales del XIX se introdujo el cultivo masivo del olivo con lo que los problemas derivados de la comercialización del aceite pasaron a primer plano: dependencia del precio establecido en los mercados internacionales, trabas a la exportación, transportes caros y malos, olvido de la administración... A pesar de no ser atendido en sus demandas al Estado y de no recibir ayuda pública, el sector privado capitalizó la producción agraria, introduciendo máquinas y prensas hidráulicas — hacia 1910, las primeras de España según el autor-, nuevos métodos de fabricación tendentes a mejorar la calidad del aceite, etc... Una modernización pues de estructuras productivas que no obstante no pudo evitar que en malas épocas, una parte importante de la población recorriere al usurero o a las cajas rurales de crédito, en manos de las personas acaudaladas, para hacer frente a los impuestos. Esto hacía que el payés tuviera, en pleno siglo XX, más confianza en el cacique que en la administración que, al fin y al cabo, no le proporcionaba ni instituciones de crédito ni ayuda ante la inestabilidad de los precios o de las cosechas. Este distrito olivarero tuvo desde Solidaridad Catalana un mismo diputado a Cortes, Francesc Macià, regionalista primero, republicano-catalanista-independiente después, futuro presidente de la Generalitat Republicana, el cual recibió siempre el favor general del distrito. $Y$ es que no en balde se convirtió en defensor ante la administración de una política de libre exportación, que era la que interesaba a productores y comerciantes de aceite.

Es difícil encontrar en los distritos electorales de la época una aquiescencia mayor entre el electorado, los caciques y el propio gobierno, que renuncia habitualmente a enviar oposición a este candidato. Sea como fuere el caso es que Francesc Maciá entendió el juego y convirtió el distrito de les Bor- 
ges y el acta de Diputado en escuela de entrenamiento político. En el distrito era un agitador político: convocaba asambleas, animaba cooperativas, promovía acciones de protesta contra la administración, a la que el año 1918 amenaza con no recoger la cosecha si no limita las trabas a la exportación del aceite. En el Congreso hacía llegar la voz de sus electores a los que defendía en sus intervenciones mucho más frecuentes que las de cualquier diputado catalán de un distrito uninominal.

Esta situación descrita no sólo nos sitúa en el tema de la modernización política, sino que también nos adentra en el problema de la movilización y desmovilización campesina. ¿Se avanzó o no en este sentido? A juzgar por los resultados electorales, poco, pero si procuramos no confundir desmovilización política con desmovilización social es posible que el payés demostrara más actividad que la que en estos momentos podemos atribuirle. El trabajo de Andreu Mayayo sobre la Conca de Barberà, otra comarca agraria de monocultivo, en este caso la vid, nos descubre la existencia de un rico movimiento cooperativista, tanto de propietarios acomodados como de pequeños agricultores, aparceros y jornaleros, surgido en respuesta a la crisis agraria de finales del XIX que arruinó las cepas de la zona vitícola de la provincia de Tarragona. Un movimiento controlado por republicanos y sindicalistas que generó conflictos, huelgas y llegó a la conquista de la admi nistración municipal de algunos municipios de la comarca antes de traspasar el siglo XX.

Igualmente nos descubre particularidades interesantes el trabajo de Joaquim Albareda y Jordi Figuerola sobre la comarca barcelonesa de Osona en donde se revela la existencia de una especie de feudalismo industrial en torno a las colonias textiles del río Ter, donde prohombres de alto espíritu emprendedor, algunos de ellos de vocación política catalanista, mantienen -con el valioso concurso de la Iglesia, de fuerte arraigo en la zona de la Plana de Vic- a los obreros y a sus familias en unas condiciones de trabajo infrahumanas, restringiendo sus más elementales derechos políticos y sindicales y, en fin, actuando con modos difícilmente superables por alguna forma de caciquismo o señoritismo rural, culpable de todos los males del país en tantos estudios generalizadores (6).

(6) Las referencias de los trabajos citados son: Andreu Mayayo, «La Conca de Barberà 18901939: de la crisi agrària a la guerra civilm, Centre d'Estudis de la Conca de Barberà, Montblanc, 1986; Gabriele Ranzato, "La aventura de una ciudad industrial. Sabadell entre el antiguo régimen y la modernidad", Nexos, Barcelona, 1987, primera edición en italiano, 1984; Francesc Sales, Les Garrigues, 1900-1936, Tesis de Licenciatura, Estudio General de Lérida (Universidad de Barcelona); Joaquim Albareda y Jordi Figuerola, "Una visión de la comar ca de Osona: anàlisi del control social en una comarca d'economia dual», a "Actituds Politiques i consol social a la Catalunya de la Restauració (1875-1923)», Lleida, 1989. 
Sin duda posteriores estudios de otras zonas ayudarán a revisar nuestras actuales concepciones sobre el problema que nos ocupa.

Pero, retomando el título general de la ponencia, podemos concluir que si desde Cataluña se impulsó con el ejemplo un cambio político neto, un nuevo sistema de relaciones entre partidos, una nueva manera de entender la acción de masas que se resolvió en algunos lugares en unas elecciones casi limpias en medio de la farsa generalizada, la burguesía catalana, supuesta depositaria de esta transformación, no quiso ni pudo estar a la altura de las circunstancias. Por un lado se encontró con un mundo rural poco evolucionado en el que se intentaba incrustar una sociedad industrial, y con unas estructuras de poder oligárquicas y caciquiles, poco dispuestas a transformarse, pero sí a pactar con quien fuera para conservar y acrecentar su poder. Por otro, los intereses de clase señalaron el límite al presumible carácter progresista -en lo económico, en lo social, en lo cultural- de esta burguesía $y$, en su prevalencia sobre todos los demás intereses, hicieron imposible un cambio real no traumático $y$, en definitiva, acabaron reorientando el proceso hacia las soluciones más autoritarias. De hecho, la burguesía barcelonesa acabó apostando por el terror blanco y la Dictadura frente al ímpetu ascendente de las organizaciones obreras, completamente ajenas al fenómeno electoral. Por ello no creo exagerado considerar que el paso a un sistema democrático de partidos acabó siendo en Cataluña un ensayo de laboratorio, desarrollado en un marco social donde la democracia burguesa era un lujo que ni los de arriba ni los de abajo pudieron permitirse, siéndole imposible al sistema en su conjunto el aprovechar esta manifestación tardía y puntual de formas democráticas para intentar reconvertirse. 